\title{
Low Energy Constants from the zero mode contribution to the pseudo-scalar correlator
}

\author{
Stanislav Shcheredin* \\ Fakultät für Physik \\ Universität Bielefeld \\ Universitätsstraße \\ D-33615 Bielefeld, Germany \\ E-mail: shcheredephysik.hu-berlin.de

\section{Wolfgang Bietenholz} \\ Institut für Physik \\ Humboldt Universität zu Berlin, \\ Newtonstr. 15 \\ D-12489 Berlin, Germany \\ E-mail: bietenho@physik.hu-berlin.de
}

We apply different types of overlap operators in quenched QCD simulations to compute the zero mode contribution to the pseudo-scalar correlator. In particular we use the conventional Neuberger Dirac operator and the overlap hypercube Dirac operator. Confronting our data with the analytical predictions by Chiral Perturbation Theory we evaluate the pion decay constant and the parameter $\alpha$ of the quenched chiral Lagrangian.

XXIIIrd International Symposium on Lattice Field Theory

25-30 July 2005

Trinity College, Dublin, Ireland

*Speaker. 


\section{Introduction}

Chiral Perturbation Theory $(\chi \mathrm{PT})$ has proven to be a valid theoretical framework for the light mesons at small energies, less than the chiral symmetry breaking scale $4 \pi F_{\pi}$, where $F_{\pi}$ is the pion decay constant. For 3-flavour QCD the mesons belong to the coset space of $U \in$ $S U(3) \otimes S U(3) / S U(3)$. The chiral Lagrangian is constructed as a hierarchical expansion in two sets of parameters, the meson momenta and the quark masses. This has to be compatible with the underlying global chiral symmetry of QCD. To render the theory a one parameter expansion one adopts a counting scheme. In the leading order, the chiral Lagrangian is parameterised by the two low energy constants (LEC) $F_{\pi}$ and $\Sigma$,

$$
\mathscr{L}^{(2)}[U]=\frac{F_{\pi}^{2}}{4} \operatorname{Tr}\left(\partial_{\mu} U \partial_{\mu} U^{\dagger}\right)-\frac{\Sigma}{2} \operatorname{Tr}\left(M U^{\dagger}+\text { h.c. }\right), \quad M=\operatorname{diag}\left(m_{u}, m_{d}, m_{s}\right),
$$

where $\Sigma$ is the chiral condensate in the chiral limit. At zero quark mass the pion decay constant is expected to be $86 \mathrm{MeV}$ [1] whereas its physical value is $93 \mathrm{MeV}$. A conventional counting scheme, where one takes the pion mass $\left(m_{\pi} \simeq \sqrt{2 m_{q} \Sigma / F_{\pi}^{2}}\right.$, with $\left.m_{q}=m_{u}=m_{d}\right)$ to be of the same order as the pion momentum, fully determines the hierarchy of the chiral expansion.

A theoretical LEC determination is only possible with the aid of ab initio QCD calculations. The aim of our ongoing project is to explore the feasibility of a LEC determination by examining different observables and lattice fermions, see also Ref. [⿰] for another contribution to these proceedings.

In a finite volume $a^{4} L^{3} \times T$ one distinguishes between two expansions of the $\chi$ PT: the socalled $p$-regime and the $\varepsilon$-regime. The $p$-regime [3] is defined in a way that the pion fits well in this box, i.e.

$$
L \gg \frac{1}{m_{\pi}} \text {. }
$$

Here one applies the conventional counting scheme to define the chiral expansion. In the $p$-regime the quantities receive finite size effects which fall off as $\exp \left(-m_{\pi} L\right)$.

In the $\varepsilon$-regime [4] one squeezes the pion into a small box and tries to calculate the resulting finite size effects. Here the following inequalities have to hold

$$
\frac{1}{4 \pi F_{\pi}} \ll L<\frac{1}{m_{\pi}} .
$$

The first condition ensures that the typical momentum in a box is much smaller than the chiral symmetry breaking scale, i.e. the expansion parameter is small. In this setting one has to change the counting scheme as

$$
\left(\frac{p}{4 \pi F_{\pi}}\right)^{2} \sim \frac{m_{\pi}}{4 \pi F_{\pi}}
$$

Since the LEC entering the expressions for the observables in the $\varepsilon$-regime are those of the infinite volume, one can obtain them by controlling the finite size effects in the $\varepsilon$-regime. In this setting one can perform simulations with extremely light quarks without the requirement of a huge volume, hence the $\varepsilon$-regime appears very appealing for lattice simulations. Unlike the $p$-regime, the observables in the $\varepsilon$-regime strongly depend on the topological sector [5]. Therefore one of the important properties of the lattice fermion formulation is that it must give a sound definition for 
the topological charge. This is fulfilled if the lattice Dirac operator $D$ obeys the Ginsparg-Wilson relation [6]

$$
\left\{D, \gamma_{5}\right\}=\frac{a}{\rho} D \gamma_{5} D .
$$

It provides us with a formulation of lattice fermions with exact lattice chiral symmetry [7]. The topological charge is identified with the index $v$ of this operator, which has exact left-handed and right-handed zero modes [8]. A particular solution to the Ginsparg-Wilson relation is given by the massless Neuberger operator [9]

$$
D_{\mathrm{ov}}=\frac{\rho}{a}\left[1+A / \sqrt{A^{\dagger} A}\right], \quad A=a \hat{D}-\rho, \quad \hat{D}=D_{\mathrm{W}},
$$

where the Wilson Dirac operator $D_{\mathrm{W}}$ is inserted into the overlap formula with a negative mass term $\rho / a$. Pursuing better locality, rotational symmetry and scaling properties, one is motivated to use a different kernel in the overlap formula [10]. As an alternative to the Wilson kernel we employed the hypercube operator [11]. Its construction is based on a truncated free perfect fermion gauged with hyperlinks. This operator has the structure

$$
D_{\mathrm{HF}}(x, y)=\sum_{\mu=1}^{4} \rho_{\mu}(x-y ; U) \gamma_{\mu}+\lambda(x-y ; U),
$$

where $x-y$ is restricted to a unit hypercube. We improved the chiral properties of this kernel by amplification factors for the couplings of the free perfect fermion and by fattening the links before constructing the hyperlinks [12]. The resulting operator is called the hypercube operator and when inserted in the overlap formula we obtain the overlap hypercube operator. Since the kernel has already good chiral properties it is expected that the overlap formula corrects it only mildly. Thus the main virtues of the truncated perfect action are preserved to a large extent.

Due to the substantial computational overhead of the overlap operator over the non-chiral operators, its applications are still mostly limited to the quenched approximation. To interpret the results of quenched QCD simulations, quenched $\chi \mathrm{PT}$ has been constructed [13]. A down side of the quenched computations is that they suffer from logarithmic volume corrections; for instance $\Sigma$ is not well defined in the infinite volume limit [14].

In this study we concentrate on a quenched lattice QCD computation of the time derivative of the zero mode contribution to the correlator of the pseudo-scalar density $P=\bar{\psi} \gamma_{5} \psi$,

$$
C_{|v|}^{\prime}(t)=\frac{d}{d t} \sum_{\vec{x}, i, j}\langle\langle j, \vec{x}, t \mid i, \vec{x}, t\rangle\langle i, 0 \mid j, 0\rangle\rangle_{v} \quad, \quad D_{\mathrm{ov}}|i, \vec{x}, t\rangle=0
$$

This quantity has been computed analytically to NNLO in $\chi \mathrm{PT}$ as well as quenched $\chi \mathrm{PT}$ in Ref. [15], which also presented a numerical study with the Neuberger operator. We extend this study to the overlap hypercube operator.

The quenched chiral Lagrangian to NNLO reads

$$
\mathscr{L}^{\mathrm{NNLO}}=\frac{F_{\pi}^{2}}{4} \operatorname{Str}\left(\partial_{\mu} U \partial_{\mu} U^{\dagger}\right)-\frac{\Sigma}{2} \operatorname{Str}(M U+\text { h.c. })-i K \Phi_{0} \operatorname{Str}(M U-\text { h.c. })+\frac{m_{0}^{2}}{2 N_{c}} \Phi_{0}^{2}+\frac{\alpha_{0}}{2 N_{c}}\left(\partial_{\mu} \Phi_{0}\right)^{2},
$$




\begin{tabular}{|l|c|c|c|c|c|c|c|}
\hline & $\rho$ & $\left\langle v^{2}\right\rangle$ & & $|v|=1$ & $|v|=2$ & $F_{\pi}[\mathrm{MeV}]$ & $\alpha$ \\
\hline overlap HF $12^{3} \times 24, \beta=5.85$ & 1 & 10.8 & 221 & 192 & $80 \pm 14$ & $-17 \pm 10$ \\
\hline Neuberger $12^{3} \times 24, \beta=5.85$ & 1.6 & 10.7 & 132 & 115 & $83 \pm 25$ & $-13 \pm 17$ \\
\hline Neuberger $16^{3} \times 32, \beta=6$ & 1.6 & 10.4 & 115 & 94 & $78 \pm 30$ & $-19 \pm 18$ \\
\hline
\end{tabular}

Table 1: Statistics and results for $F_{\pi}$ and $\alpha$ at fitting range $s_{\max }=1$.

where $U \in S U\left(N_{f} \mid N_{f}\right) \otimes S U\left(N_{f} \mid N_{f}\right) / S U\left(N_{f} \mid N_{f}\right)$ and "Str" is the corresponding "supertrace". $\mathscr{L}^{\mathrm{NNLO}}$ contains now five quenched LEC: $F_{\pi}, \Sigma, K, m_{0}, \alpha_{0}$. The peculiarity of the quenched chiral Lagrangian is the presence of a scalar field $\Phi_{0}$, which is responsible for the quenching effects.

We consider only the first term in the Taylor expansion of $C_{|v|}^{\prime}(t)$ about $T / 2$. In a box $L^{3} \times T$ it takes the form [15]

$$
C_{|v|}^{\prime}(t) / L^{2}=D_{|v|}^{\mathrm{NNLO}}\left(F_{\pi},\left\langle v^{2}\right\rangle, \alpha\right) \frac{s}{T}+\mathscr{O}\left(\left(\frac{s}{T}\right)^{3}\right), \quad s=t-T / 2
$$

where $\alpha$ is defined by $\alpha / 2 N_{c}:=\alpha_{0} / 2 N_{c}-2 K F_{\pi} / \Sigma$, and the dependence on $\left\langle v^{2}\right\rangle$ originates from the Witten-Veneziano formula [16].

\section{Numerical setup and results}

We performed quenched QCD simulations with the Wilson gauge action at $\beta=5.85$ on a lattice of size $12^{3} \times 24$, and at $\beta=6$ on $16^{3} \times 32$. The parameters for the overlap operators are described in Ref. [17]. In both cases, the physical volume amounts to $(1.48 \mathrm{fm})^{3} \times 2.96 \mathrm{fm}$. We chose it large enough, referring to the experience with the chiral Random Matrix Theory [18] and to the behaviour of the axial correlators in the $\varepsilon$-regime [19]. We computed zero eigenmodes for the overlap hypercube operator at $\beta=5.85$ and for the Neuberger operator on both lattices. Our statistics is collected in Table 1. We fixed the value of $\left\langle v^{2}\right\rangle$ from our simulations and performed a combined two parameter fit of the leading term in Eq. (1.10) to our data in the topological sectors $|v|=1,2$ in intervals $s \in\left[-s_{\max }, s_{\max }\right]$, with $s_{\max }=1 \ldots 3$. Preliminary results were presented in Ref. [17], and our new results are given in Figure 1 and in Table 1. Our values for $F_{\pi}$ and $\alpha$ are a little lower than the results reported in Ref. [15] (for the Neuberger operator in isotropic boxes). ${ }^{1}$

We still have sizable (jackknife) error bars. We see, however, that our values of $F_{\pi}$ and $\alpha$ are compatible within the errors for different overlap fermions and for two lattice spacings. In particular the result with the overlap hypercube operator favours a negative value for the parameter $\alpha$. A negative value has also been obtained for the "original" parameter $\alpha_{0}$ from the full pseudoscalar correlator [20].

\section{Conclusions and outlook}

We computed quenched values of $F_{\pi}$ and the parameter $\alpha$ from the zero mode contribution to the pseudo-scalar correlator using the Neuberger and the overlap hypercube operator in the chiral

\footnotetext{
${ }^{1} \mathrm{~A}$ different way to compute $F_{\pi}$ in the $\varepsilon$-regime is to use the axial-vector correlator [2, 19, 20]. That method suggests a somewhat larger value of $F_{\pi}$ (at finite $m_{q}$ ).
} 

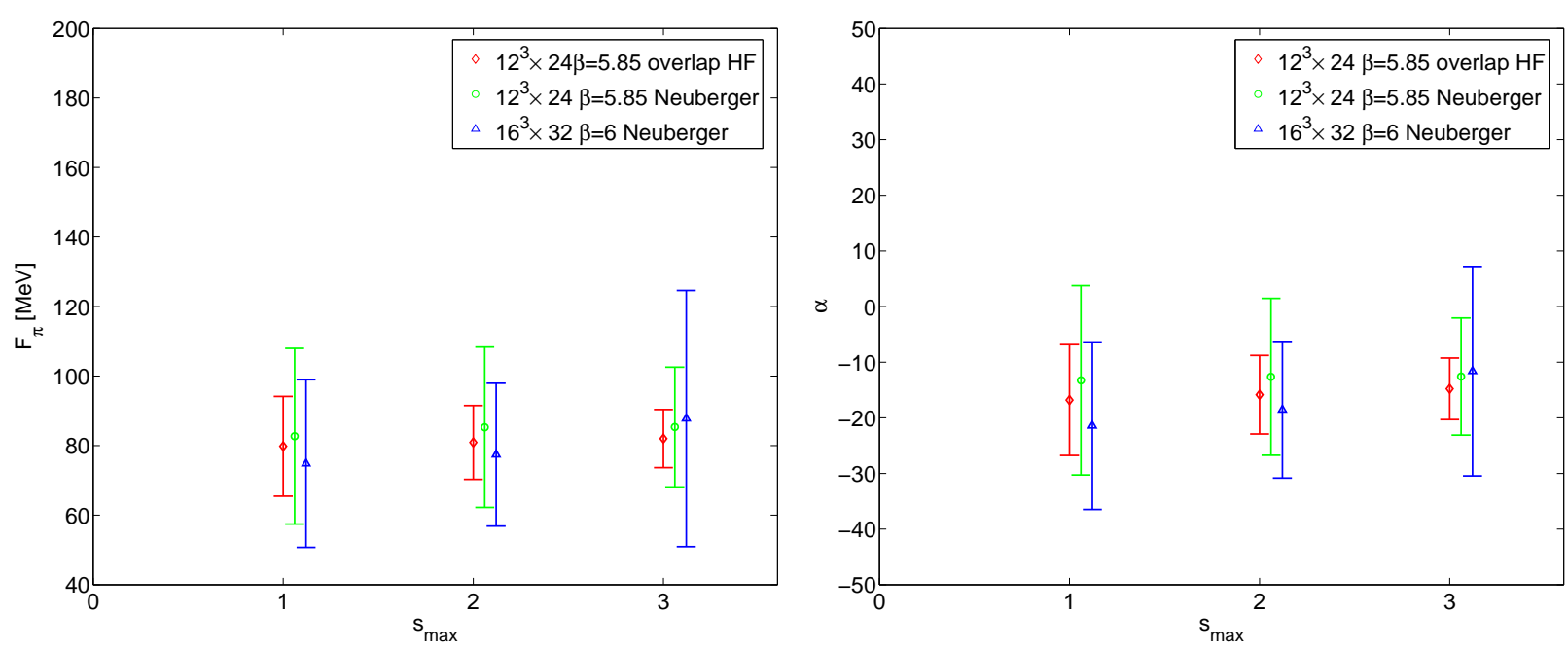

Figure 1: $F_{\pi}$ and $\alpha$ according to Eq. (1.10) vs. the width of the fitting range $s_{\max }$.

limit, in a volume $(1.48 \mathrm{fm})^{3} \times 2.96 \mathrm{fm}$. The results are compatible for two lattice spacings and different overlap operators within the errors. Our result for $F_{\pi}$ obtained with this method (in the given volume) is close to the phenomenological value (in the chiral limit). It would be interesting to extend this study to a finer lattice spacing for the overlap HF and to increase our statistics.

\section{Acknowledgments}

We would like to thank M. Papinutto and C. Urbach for providing us with powerful eigenvalue routines. We acknowledge discussions with S. Dürr, K.-I. Nagai, H. Stüben and P. Weisz. This work is supported by the Deutsche Forschungsgemeinschaft through SFB/TR9-03. The computations have been performed on the IBM p690 clusters of the HLRN "Norddeutscher Verbund für Hochund Höchstleistungsrechnen" (HLRN) and at NIC, Forschungszentrum Jülich.

\section{References}

[1] G. Colangelo and S. Dürr, The pion mass in finite volume, Eur. Phys. J. C 33 (2003) 543 [hep-lat/0311023].

[2] W. Bietenholz and S. Shcheredin, Overlap hypercube fermions in QCD with light quarks, hep-lat/0508016.

[3] J. Gasser and H. Leutwyler, Light quarks at low temperatures, Phys. Lett. B 184 (1987) 83.

[4] J. Gasser and H. Leutwyler, Thermodynamics of chiral symmetry, Phys. Lett. B 188 (1987) 477.

[5] H. Leutwyler and A. Smilga, Spectrum of Dirac operator and role of winding number in QCD, Phys. Rev. D 46 (1992) 5607.

[6] P. H. Ginsparg and K. G. Wilson, A remnant of chiral symmetry on the lattice, Phys. Rev. D 25 (1982) 2649.

[7] M. Lüscher, Exact chiral symmetry on the lattice and the Ginsparg-Wilson relation, Phys. Lett. B 428 (1998) 342 [hep-lat/9802011]. 
[8] P. Hasenfratz, V. Laliena and F. Niedermayer, The index theorem in QCD with a finite cut-off, Phys. Lett. B 427 (1998) 125 [hep-lat/9801021] .

[9] Y. Kikukawa and H. Neuberger, Overlap in odd dimensions, Nucl. Phys. B 513 (1997) 735 [hep-lat/9707016]. H. Neuberger, Exactly massless quarks on the lattice, Phys. Lett. B 417 (1998) 141 [hep-lat/9707022]. H. Neuberger, More about exactly massless quarks on the lattice, Phys. Lett. B 427 (1998) 353 [hep-lat/9801031].

[10] W. Bietenholz, Solutions of the Ginsparg-Wilson relation and improved domain wall fermions, Eur. Phys. J. C 6 (1999) 537 [hep-lat/9803023].

[11] W. Bietenholz and U. J. Wiese, Perfect lattice actions for quarks and gluons, Nucl. Phys. B 464 (1996) 319 [hep-lat/ 9510026 ]. W. Bietenholz, R. Brower, S. Chandrasekharan and U. J. Wiese, Progress on perfect lattice actions for QCD, Nucl. Phys. B (Proc. Suppl.) 53 (1997) 921

[hep-lat/9608068]. W. Bietenholz, N. Eicker, A. Frommer, Th. Lippert, B. Medeke, K. Schilling and G. Weuffen, Preconditioning of improved and 'perfect' fermion actions, Comput. Phys. Commun. 119 (1999) 1 [hep-lat/9807013].

[12] W. Bietenholz, Convergence rate and locality of improved overlap fermions, Nucl. Phys. B 644 (2002) 223 [hep-lat/0204016].

[13] C. W. Bernard and M. F. L. Golterman, Chiral perturbation theory for the quenched approximation of QCD, Phys. Rev. D 46 (1992) 853 [hep-lat/92 04007 ]. P. H. Damgaard, M. C. Diamantini, P. Hernández and K. Jansen, Finite-size scaling of meson propagators, Nucl. Phys. B 629 (2002) 445 [hep-lat/ 0112016$]$. P. H. Damgaard, P. Hernández, K. Jansen, M. Laine and L. Lellouch, Finite-size scaling of vector and axial current correlators, Nucl. Phys. B 656 (2003) 226 [hep-lat/0211020].

[14] P. H. Damgaard, Quenched finite volume logarithms, Nucl. Phys. B 608 (2001) 162 [hep-lat/0105010].

[15] L. Giusti, P. Hernández, M. Laine, P. Weisz and H. Wittig, Low energy couplings of QCD from topological zero-mode wave functions, JHEP 0401 (2004) (003) [hep-lat / 0312012 ]. P. Hernández et al., these Proc.

[16] E. Witten, Current algebra theorems for the U(1) 'Goldstone boson', Nucl. Phys. B 156 (1979) 269. G. Veneziano, U(1) without instantons, Nucl. Phys. B 159 (1979) 213.

[17] S. Shcheredin, Simulations of lattice fermions with chiral symmetry in quantum chromodynamics, Ph.D. thesis (Berlin, 2004) [hep-lat/0502001]. W. Bietenholz and S. Shcheredin, Relating chiral perturbation theory and QCD simulations with overlap hypercube fermions, hep-lat/0502010.

[18] W. Bietenholz, K. Jansen and S. Shcheredin, Spectral properties of the overlap Dirac operator in QCD, JHEP 07 (2003) 033 [hep-lat/0306022]. L. Giusti, M. Lüscher, P. Weisz and H. Wittig, Lattice QCD in the epsilon regime and Random Matrix Theory, JHEP 11 (2003) 023 [hep-lat/0309189]. QCDSF-UKQCD Collaboration, Quark spectra and light hadron phenomenology from overlap fermions with improved gauge field action, Nucl. Phys. B (Proc. Suppl.) 129 \& 130 (2004) 456 [hep-lat / 0310028$].$

[19] W. Bietenholz, T. Chiarappa, K. Jansen, K.-I. Nagai and S. Shcheredin, Axial correlation functions in the epsilon regime: a numerical study with overlap fermions, JHEP 0402 (2004) 023 [hep-lat/0311012].

[20] H. Fukaya, S. Hashimoto and K. Ogawa, Low-lying mode contribution to the quenched meson correlators in the epsilon-regime, hep-lat/0504018. K. Ogawa et al., these Proc. 